\title{
LA PRENSA HISTÓRICA COMO HERRAMIENTA DE RECOPILACIÓN DE INFORMACIÓN METEOROLÓGICA Y CLIMÁTICA. EL CASO DE LA CIUDAD DE TARRAGONA (ESPAÑA)
}

\author{
Ricard Ripoll Pi ${ }^{\mathrm{a}}$, Marc Prohom Duran ${ }^{\mathrm{ab}}$, Juan Carlos Peña Rabadán ${ }^{\mathrm{ab}}$, y Javier Martín Vide ${ }^{\mathrm{b}}$ \\ Servei Meteorològic de Catalunya. Àrea de Climatologia ${ }^{a}$ \\ Grup de Climatologia. Universitat de Barcelona ${ }^{\mathrm{b}}$
}

\section{RESUMEN}

La prensa histórica es una fuente de información meteorológica y climática adicional a la fuente habitual de los archivos meteorológicos. En este estudio se presenta el proceso de recuperación de los valores de las variables meteorológicas de la ciudad de Tarragona (Spain) a partir de periódicos antiguos. En primer lugar se detalla la fase de localización de los datos meteorológicos, dispersos en diferentes fondos, datos que han sido digitalizados manualmente para su preservación y posterior manipulación. El análisis de las distintas variables recuperadas permite a menudo observar los diferentes cambios en las mediciones, las localizaciones y los instrumentos utilizados. Así pues, se dispone de una recuperación de metadatos para que la posterior manipulación de las series climáticas sea lo más homogénea posible. Finalmente se muestran las potencialidades y carencias de la recuperación de datos meteorológicos a través de la prensa antigua.

Palabras clave: prensa histórica, climatología histórica, Tarragona, fuentes documentales, variabilidad climática.

\section{ABSTRACT}

Historical press as a meteorological and climatic information recovery source. The case of Tarragona city (Spain)

The historical press is a source of meteorological and climatic information in addition to the traditional documentary sources. In this study is presented the recovery process of meteorological variables from the city of Tarragona (Spain) from old newspapers. Firstly, it details the process of locating data in digital form, scattered in different journals, data that has been manually digitized for preservation and subsequent handling. The analysis of different meteorological variables recovered can often see the different changes in measurements, locations and instruments used. It has metadata retrieval for subsequent manipulation of weather variables is as homogeneous as possible. Finally we show the strengths and weaknesses of meteorological data retrieval through old press.

Key words: historical press, historical climatology, Tarragona, documentary sources, climate variability.

\section{INTRODUCCIÓN}

Durante las últimas décadas la climatología ha experimentado grandes avances científicos y técnicos, gracias en buena medida a la creciente preocupación por el cambio climático y sus posibles consecuencias. Estos avances en Europa han proporcionado a la ciencia del clima una gran tecnificación en diferentes ámbitos, que hacen que la climatología histórica tenga un gran potencial (Brázdil et al, 2005). De esta manera, la informática ha hecho posible que se puedan crear modelos de circulación atmosférica muy fiables a pocos días vista, y que permiten a su vez, poder hacer una buena predicción meteorológica. Los

Contacto: Ricard Ripoll Pi; ricardripi@gmail.com; Marc Prohom Duran: mprohom@meteo.cat; Juan Carlos Peña Rabadán: jpena@meteo.cat; Javier Martín Vide: jmartinvide@ub.edu 
avances y una utilización cada vez más popular de los recursos informáticos han permitido también el estudio de un gran número de series de datos instrumentales, que hasta hace pocos años tenían grandes limitaciones en su tratamiento, tanto en controles de calidad y homogeneización, como en su posterior tratamiento (Barriendos et al., 1997). Así pues, es ahora el momento de recuperar las series de datos meteorológicos antiguos para poder tratar climáticamente los comportamientos de las diferentes variables meteorológicas en un lugar concreto.

En la actualidad, los registros paleoclimáticos han adquirido un gran interés, ayudando así a conseguir proxy data, que permiten reconstruir el clima del pasado con precisión. La climatología histórica es una especialidad paleoclimática que obtiene información exclusivamente de fuentes documentales históricas (Barriendos, 1999). Entre los documentos que pueden servir de fuente de datos, encontramos fondos muy variados, como por ejemplo: archivos notariales, judiciales, fiscales, administrativos, económicos, militares, privados, etc, donde destacan las crónicas, las actas municipales, las actas eclesiásticas (López Cordero, 2006), los diarios de navegación y cuadernos de bitácora (Prohom, 2002), entre muchos otros.

Los estudios de climatología histórica en Europa han permitido reconstruir el clima de las últimas centurias a diferentes escalas espaciales, destacando aquellos que abarcan todo el continente (Pauling et al, 2006), y diferentes análisis centrados en áreas concretas, como, por ejemplo, los Alpes (Casty et al, 2005), el Mediterráneo Occidental (Barriendos y Llasat, 2003), países concretos, como Noruega (Nordli, 2001) o Polonia (Przybylak et al, 2001), o subregiones (Rodrigo et al, 1998) (Piervitali y Colacino, 2001) (Domínguez-Castro et al, 2008).

En Catalunya la climatología histórica se ha utilizado para la reconstrucción de episodios catastróficos (Thorndycraft et al, 2006), y también para la evaluación de la variabilidad climática (Barriendos, 19961997) (Martín-Vide y Barriendos, 1995) (Barriendos, 2005).

La prensa es una fuente de información meteorológica adicional a las fuentes tradicionales (servicios meteorológicos o instituciones privadas) que puede ser de gran interés climático (Llasat et al, 2009). En los últimos años muchos archivos comarcales y locales han escaneado y almacenado digitalmente las publicaciones antiguas de prensa histórica y las han puesto a disposición de la ciudadanía a través de la red de internet (Prohom y Herrero, 2008). Se han realizado algunos ensayos para utilizar la prensa histórica como herramienta de información meteorológica en Andalucía (Fernández-Montes y Rodrigo, 2010), pero todavía ninguno como el que presentamos a continuación. En el presente trabajo se presenta la digitalización de los datos meteorológicos diarios disponibles en las publicaciones que contenían información meteorológica de la ciudad de Tarragona, para después proceder a la validación de la fuente de información meteorológica. Así, se ha efectuado un vaciado exhaustivo de la información meteorológica publicada en la prensa antigua de la ciudad de Tarragona entre los años 1809 y 1944, para enlazar con la serie de datos oficiales existente en la actualidad, disponible a partir de 1930, en el caso de los datos diarios, y de 1903, en el caso de los mensuales.

Tarragona es una ciudad situada en la costa sur catalana, en el Mediterráneo Occidental, en la margen izquierda de la desembocadura del río Francolí. Actualmente es capital de provincia con una extensión de 65,2 km² y una población de 133.954 habitantes, según el Padrón municipal de 2012. El clima típico de Tarragona es el Mediterráneo Catalán del Litoral Sur (Martín-Vide, 1992), con una distribución de la precipitación irregular y unos totales anuales modestos. La estación más lluviosa es el otoño y la más seca, el invierno. El período árido comprende los meses de junio y julio, si bien encontramos también un mínimo pluviométrico secundario en febrero. La precipitación media anual se sitúa en torno a los 500 mm o algo más. El régimen térmico se caracteriza por veranos calurosos e inviernos templados, con una temperatura media anual de cerca de $17^{\circ} \mathrm{C}$. Las heladas son poco frecuentes, concentradas en los tres meses de invierno.

\section{IDENTIFICACIÓN DE LOS DATOS Y METODOLOGÍA}

Para la realización del estudio se han utilizado los datos meteorológicos disponibles en la prensa escrita de Tarragona, escaneados y almacenados en formato digital desde el año 1809 hasta 1944 (Tabla 1). Los datos meteorológicos publicados en cada periódico se han digitalizado manualmente, para proceder después a un análisis de los datos. 
Esta digitalización se ha podido hacer gracias a los ejemplares de prensa escaneada que están disponibles en la web de la Biblioteca-Hemeroteca Municipal de Tarragona.

La recuperación de todas las variables meteorológicas disponibles en la prensa se ha realizado mediante la digitalización de todos los parámetros que aparecían publicados. El proceso de digitalización ha topado con algunas dificultades, como por ejemplo desconocer la longitud exacta del período de cada punto de observación, así como la exacta localización de estos puntos de observación (algunos de los cuales no ha sido posible ubicar). Pero también encontramos cambios en el instrumental y en las unidades de medida de las variables, así como en el número de las observaciones diarias, entre otros problemas fruto del tratamiento de datos antiguos (Prohom et al., 2012).

Tablal. Disponibilidad de observaciones según período y fuente en Tarragona

\begin{tabular}{|c|c|c|c|c|}
\hline Período & Fuente de los datos & Variables publicadas $_{1}$ & $\begin{array}{c}\text { Horas de } \\
\text { observación }\end{array}$ & Ubicación \\
\hline $1809-1811$ & Diario de Tarragona $1^{a}$ época & $\mathrm{P}, \mathrm{T}, \mathrm{DV}, \mathrm{A}$ & 06h-12h-18h & desconocido \\
\hline $1844-1846$ & El Faro del Francoli & $\mathrm{P}, \mathrm{T}, \mathrm{DV}, \mathrm{A}$ & 07h-12h-17h & desconocido \\
\hline $1856-1859$ & $\begin{array}{l}\text { Diario Mercantil de Avisos y } \\
\text { Noticias }\end{array}$ & P, T, DV, A & 06h-12h-18h & desconocido \\
\hline 1879-1881 & Diario de Tarragona $2^{a}$ época & $\begin{array}{c}\text { P, Tm, Tx, Tn, HR, Ts, Th, Ev, DV, VV, A, } \\
\text { PPT }\end{array}$ & - & $\begin{array}{l}\text { Instituto provincial de } \\
\text { Tarragona }\end{array}$ \\
\hline $1886-1887$ & El Orden & P, Tx, Tn, Ts, Dv, VV, A, PPT, Ev & $09 \mathrm{~h}-15 \mathrm{~h}$ & $\begin{array}{l}\text { Instituto provincial de } \\
\text { Tarragona }\end{array}$ \\
\hline $1889-1890$ & El Mercantil & P, Tx, Tn, Ts, Th, Dv, VV, A, PPT, Ev & $09 \mathrm{~h}-15 \mathrm{~h}$ & $\begin{array}{c}\text { Estación Vitícola y } \\
\text { Enológica de Tarragona }\end{array}$ \\
\hline $1889-1890$ & La Provincia & P, Tx, Tn, Ts, Th, Dv, VV, A, PPT, Ev & $09 \mathrm{~h}-15 \mathrm{~h}$ & $\begin{array}{c}\text { Estación Vitícola y } \\
\text { Enológica de Tarragona }\end{array}$ \\
\hline 1890 & El Pabellón Liberal & P, Tx, Tn, Ts, Th, Dv, VV, A, PPT, Ev & $09 \mathrm{~h} 15 \mathrm{~h}$ & $\begin{array}{c}\text { Estación Vitícola y } \\
\text { Enológica de Tarragona }\end{array}$ \\
\hline 1890 & La Opinión & P, Tx, Tn, Ts, Th, Dv, VV, A, PPT, Ev & $09 \mathrm{~h}-15 \mathrm{~h}$ & $\begin{array}{c}\text { Estación Vitícola y } \\
\text { Enológica de Tarragona }\end{array}$ \\
\hline $1897-1898$ & El Comercio & P, Tx, Tn, Tm, DV & $09 \mathrm{~h}-15 \mathrm{~h}$ & $\begin{array}{l}\text { Instituto provincial de } \\
\text { Tarragona }\end{array}$ \\
\hline 1903 & Diario de Tarragona $3^{a}$ época & P, Tx, Tn, DV, VV, A, HR, PPT & 09h-15h & $\begin{array}{c}\text { Universidad Pontificia de } \\
\text { Tarragona } \\
\end{array}$ \\
\hline $1916-1927$ & Diario de Tarragona $3^{a}$ época & P, Tx, Tn, DV, VV, A, HR, PPT & $09 \mathrm{~h}-15 \mathrm{~h}$ & $\begin{array}{l}\text { Universidad Pontificia } \\
\text { de Tarragona e Instituto } \\
\text { provincial de Tarragona }\end{array}$ \\
\hline $1920-1926$ & Tarragona & P, Tx, Tn, DV, VV, A, HR, PPT & $09 \mathrm{~h}-15 \mathrm{~h}$ & $\begin{array}{l}\text { Instituto provincial de } \\
\text { Tarragona }\end{array}$ \\
\hline $1927-1937$ & Diario de Tarragona $3^{a}$ época & P, Tx, Tn, DV, VV, A, HR, PPT & 08h-18h & $\begin{array}{l}\text { Instituto provincial de } \\
\text { Tarragona }\end{array}$ \\
\hline $1930-1931$ & La Voz de la Provincia & P, Tx, Tn, DV, VV, A, HR, PPT & 08h-18h & $\begin{array}{l}\text { Instituto provincial de } \\
\text { Tarragona }\end{array}$ \\
\hline $1936-1937$ & Llibertat & P, Tx, Tn, DV, VV, A, HR, PPT & $08 \mathrm{~h}-18 \mathrm{~h}$ & $\begin{array}{l}\text { Instituto provincial de } \\
\text { Tarragona }\end{array}$ \\
\hline $1942-1944$ & Diario Español & P, Tx, Tn, Ts, HR, DV, VV, A, PPT, VIS & $19 \mathrm{~h}$ & - \\
\hline
\end{tabular}

1 P: Presión atmosférica, T: Temperatura, Tx: Temperatura Máxima, Tn: Temperatura Mínima, Ts: Temperatura Termómetro Seco, Th: Temperatura Termómetro Húmedo, Tm: Temperatura Media, HR: Humedad Relativa, Ev: Evaporación, DV: Dirección del Viento, VV: Velocidad del Viento, A: Estado del Cielo, VIS: Visibilidad, PPT: Precipitación

Fuente: Elaboración propia

Así pues, una vez enumerada la prensa escrita digitalizada en la que se puede encontrar información meteorológica de la ciudad de Tarragona, se procede a hacer un análisis variable por variable, con el objetivo de valorar la calidad de los datos y evaluar si la información meteorológica procedente de los antiguos rotativos constituye una buena fuente de datos para el análisis climático. 


\section{ANÁLISIS DE LAS VARIABLES METEOROLÓGICAS}

Cada una de las variables meteorológicas presenta diferencias a lo largo de la serie de datos recuperada, destacando, entre otros aspectos, los periodos de observación (en algunos casos hay variables que dejan de publicarse durante algún momento concreto) o las horas de observación.

\subsection{Distribución temporal}

El espacio temporal para el que se han recuperado los datos meteorológicos empieza en el año 1809 y llega hasta 1944, tal y como se muestra en Figura 2, donde se señalan los años en que se dispone de información meteorológica, junto con el porcentaje de datos por anual recuperado.

La Figura 2 indica un gran número de años durante el período 1809-1944 en los que no se dispone de datos meteorológicos. Al inicio de la serie existen casi 3 años seguidos de datos meteorológicos gracias al Diario de Tarragona, que empezó a publicar las observaciones meteorológicas en su edición diaria. A partir de 1811 y hasta 1855 nos encontramos con un largo periodo de tiempo en el que no se publican datos meteorológicos. A partir de 1856 y hasta 1915 aparecen distintos periódicos que proporcionan la información meteorológica, aunque no con la suficiente cadencia como para disponer de una buena serie de datos, ya que durante este tiempo los rotativos que publicaban las observaciones meteorológicas no sobrepasaban los 2 o 3 años seguidos de difusión.

Finalmente se dispone de un período de datos bastante continuado, con los datos meteorológicos diarios desde 1916 hasta el final de la serie el año 1944, donde encontramos solamente un vacío de datos entre los años 1938 y 1940, periodo que coincide con la Guerra Civil Española. Durante este largo lapso temporal se dispone de numerosos años completos a resolución diaria, entendiendo por años completos aquellos en los que cada mes dispone de más del 80\% de observaciones diarias. Es conveniente remarcar que durante los fines de semana y festivos, la prensa comarcal de Tarragona no publicaba sus números, y, al no disponer de publicaciones, no se han podido recuperar los datos meteorológicos diarios de estos días a través de estas fuentes escritas.

Figura 2. Porcentaje anual de los datos meteorológicos disponibles entre los años 1809 y 1944.

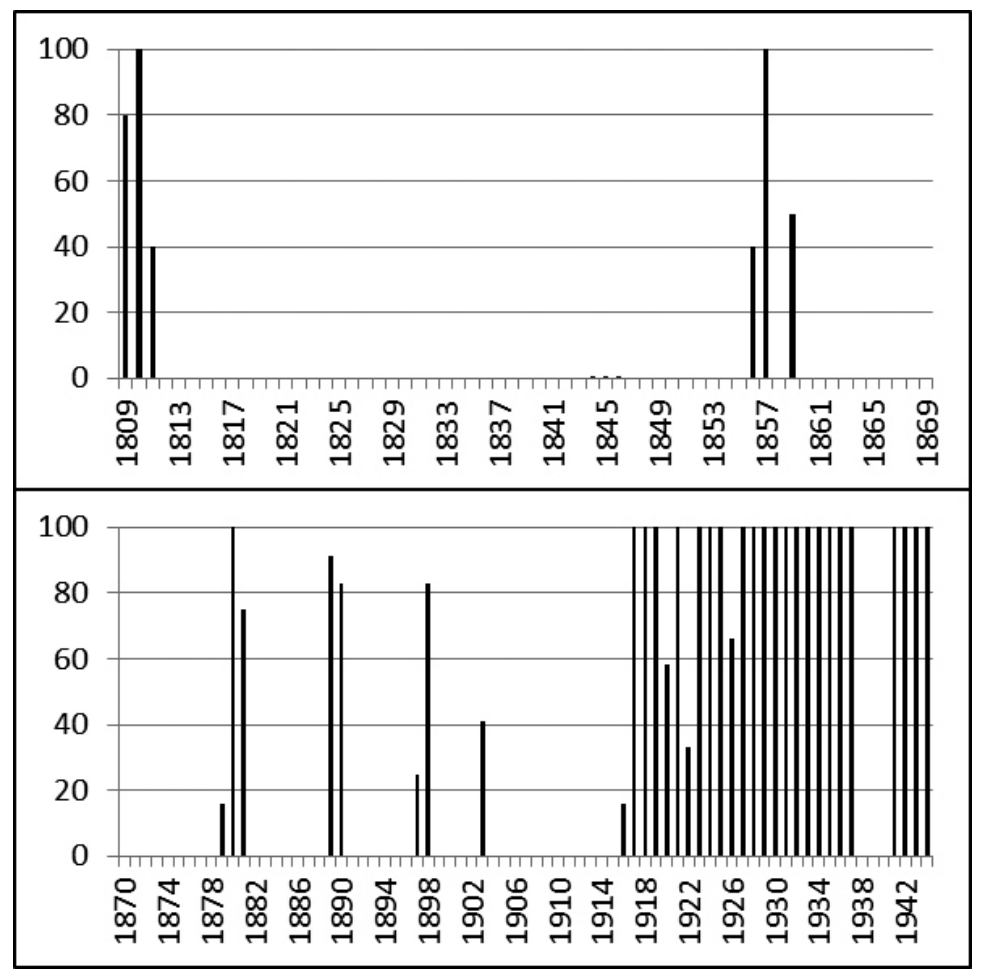

Fuente: Elaboración propia 


\subsection{La temperatura}

La temperatura se ha medido de diferentes maneras. En general, desde los inicios de la serie en 1809 hasta 1856 la temperatura se medía en grados Reamur $\left({ }^{\circ} \mathrm{R}\right)$, y es a partir de 1856 hasta el final de la serie en 1944 que los datos de temperatura ya se proporcionan en grados Celsius $\left({ }^{\circ} \mathrm{C}\right)$.

También hay un gran número de observaciones horarias a diferentes momentos del día (Tabla 1), pero generalmente se hacían tres observaciones: una por la mañana (06 o 07h), al mediodía ( $12 \mathrm{ol} h \mathrm{~h})$ y otra por la tarde/noche (17, 18 o 19h). También encontramos un par de épocas, entre los años 1879-1881 y 1897-1898, en que sólo se indica la temperatura media del día en concreto, junto con la temperatura máxima (Tx) y la temperatura mínima (Tn).

Las temperaturas máximas y mínimas merecen una mención especial, por la importancia que tienen en estudios de cambio climático. Estas observaciones empiezan a aparecer a partir del año 1879 con la $2^{a}$ época del Diario de Tarragona, cuando la información meteorológica publicada procede del Instituto provincial de Tarragona (Tabla 1). Los registros de Tx y Tn aparecen desde 1879 en ${ }^{\circ} \mathrm{C}$, hasta el final de la serie en 1944, incluso cuando hubo un cambio en el origen de los datos. Así pues, la Estación Vitícola y Enológica de Tarragona, la Universidad Pontificia de Tarragona y el Instituto provincial de Tarragona siempre proporcionaron los datos de Tx y Tn.

La descripción de la ubicación de los termómetros no la encontramos hasta 1889 en la Estación Vitícola y Enológica de Tarragona (Figura 3), donde se especifica que los datos se toman a 1,50 metros del suelo y a la sombra, y también la máxima al sol y la mínima a cielo descubierto. Todas ellas en ${ }^{\circ} \mathrm{C}$.

Figura 3. Ejemplo de información referente a las temperaturas máxima y mínima registradas en la Estación Vitícola y Enológica de Tarragona.

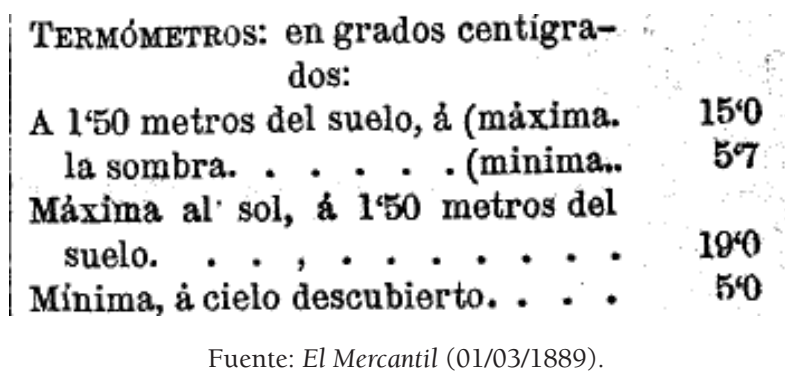

Figura 4. Ejemplo de información referente a las temperaturas máxima y mínima registradas en la Universidad Pontificia de Tarragona.

Temperaturas en grados centgr.-A las 9, nor-

mal 10 60; á las 3, 14'0. Máxima al sol, 20; som-

bra, 16. Minima, reflec. 350; sombra, 7.0.

Fuente: Diario de Tarragona (10/01/1903).

Figura 5. Ejemplo de información referente a las temperaturas máxima y mínima registradas en el Instituto Provincial de Tarragona.

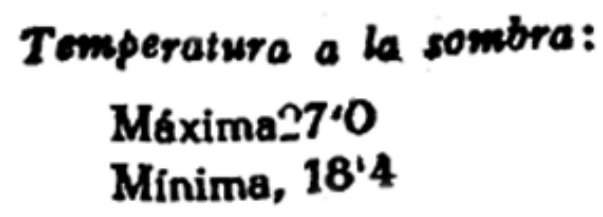

Temperatura a l'ombra: Màxima, 27'5, I mínima, $19: 5$.

Fuente: La Voz de la Provincia (02/09/1930) y La Llibertat (02/09/1936).

En la Universidad Pontificia las temperaturas máxima y mínimas se expresaban en ${ }^{\circ} \mathrm{C}$ y se tomaban a la sombra y al sol, tal y como se puede observar en la Figura 4. En cambio, en este caso no se indica la altura sobre el suelo a la cual se encontraba el termómetro. 
Por lo que se refiere a los datos obtenidos del observatorio del Instituto Provincial de Tarragona, no es hasta el año 1930 en el diario La Voz de la Provincia que se describe que las temperaturas máximas y mínimas son a la sombra, cosa que más tarde corrobora el diario La Llibertat (Figura 5).

\subsection{La precipitación}

La precipitación (PPT) es, juntamente con la Tx y la Tn, la variable meteorológica más importante a la hora de realizar estudios de cambio climático. Como en el caso de las Tx y Tn, los valores de PPT empiezan a aparecer a partir de 1879, con la publicación de los datos meteorológicos en la $2^{a}$ época del Diario de Tarragona, cuando los registros originales proceden del Instituto Provincial de Tarragona.

La medida que se utiliza para la PPT son los milímetros ya desde 1879 (Figura 6), por lo que no es necesario realizar cambio de unidades alguno.

El problema más importante que presenta la precipitación estriba en que si un día no se publicó el diario, y en ese día hubo lluvia, la carencia de registro afecta a los totales mensuales y anuales de precipitación.

Figura 6. Ejemplo de observaciones del observatorio del Instituto Provincial de Tarragona.

\section{OBSERVACIONES METEOROLOGICAS.}

$$
\text { Instituto de Tarragona.-Dia } 9 .
$$

Barómetro mercurio, 769 milímetros.-Temperatura media ordinaria $5 ‘ 83$ grados. - Id. máxima ;6.-Id. minima 0'25.-Higrómetro Sausure 3̋0'00.-Psıcrómetro: termómetro húmedo 3.Id. id. seco 2'83.-Evaporímetro 0 mils.-Direccion del viento, NO.-Velocidad del viento 0 metros.-Nubes 0.-Pluvimetro 0 míls.

Fuente: Diario de Tarragona (10/12/1879).

\subsection{La presión atmosférica}

La presión atmosférica (P) es una de las variables meteorológicas que se anota y se publica desde el inicio de la serie de datos recuperada. Por tanto, se dispone de datos de presión atmosférica desde 1809 cuando la medida de la variable era en pulgadas y líneas probablemente de París (Barriendos et al., 1997, Figura 7). Es a partir de 1879 cuando las medidas pasan a expresarse en milímetros de mercurio ( $\mathrm{mmHg}$ ), coincidiendo con las publicaciones del Diario de Tarragona 2a época (Figura 6).

Figura 7. Ejemplo de observaciones de presión atmosférica en pulgadas y líneas probablemente de París.

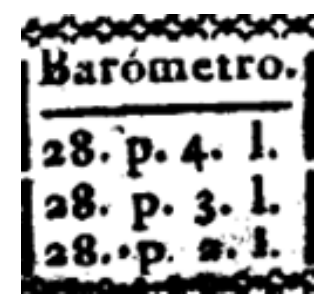

Fuente: Diario de Tarragona (21/02/1809)

En el caso de la presión atmosférica nos encontramos, tal como pasaba con la variable temperatura, que durante algunos períodos existieron tres observaciones diarias, mientras que en otros períodos sólo se dispone de una observación diaria. Por norma general, si existe la medida de la temperatura a una hora, también hay la correspondiente observación de presión atmosférica, a excepción de los promedios 
diarios y los extremos máximos y mínimos, que sólo se publicaban de la variable temperatura y no con la presión atmosférica.

\subsection{La humedad}

Esta variable merece algunas consideraciones especiales. La variable humedad relativa (HR) no se empieza a publicar como tal hasta el año 1903, coincidiendo con el retorno de la publicación de las observaciones meteorológicas del Diario de Tarragona en su $3^{a}$ época. En el año 1903 las unidades estaban en tanto por 1 , y a partir de 1916 los datos de humedad relativa se encuentran en tanto por 100 (\%) (Figura 8).

Figura 8. Ejemplo de observaciones de Humedad Relativa.

\section{Humedad relaliza.-A las 9, 0.86. A las 3, 0'89. \\ Humedad relativa, mañana, 84.-Id. tarde, 77.-Evaporación, 0'90.}

Fuente: Diario de Tarragona (10/01/1903) en tanto por 1, y (09/02/1917) en tantos por 100.

En períodos anteriores a 1903 y desde el año 1879 se publicaban los datos de temperaturas del termómetro seco (Ts) y del termómetro húmedo (Th), a partir de los cuales se puede derivar la humedad relativa correspondiente de la observación gracias a la tablas psicométricas, que dan un valor de humedad relativa dependiendo de la diferencia de temperatura entre Ts y Th. Así pues, se pueden recuperar datos de humedad relativa desde 1879, para diferentes horas de observación.

\subsection{La evaporación}

Entre 1809 y 1879 no se dispone de datos de evaporación (Ev). La evaporación empieza a medirse y publicarse a partir de 1879 con los datos extraídos del Diario de Tarragona $2^{a}$ época hasta el año 1881. Después, la evaporación no vuelve a aparecer hasta el año 1916 con las nuevas publicaciones del Diario de Tarragona en su $3^{a}$ época, y hasta 1937. Esta variable meteorológica solamente se publica en el Diario de Tarragona, Tarragona, y La Voz de la Provincia. No se dispone de metadato que indique cuál era el aparato que se utilizaba para la medida ni cuáles eran las unidades utilizadas, lo que hace pensar que se trate de milímetros de agua evaporada durante el día, ya que la resolución de los datos es diaria.

\subsection{Dirección y velocidad del viento}

En los inicios de la serie, en el año 1809 sólo se indica la dirección del viento (DV). Mediante los cuatro puntos cardinales y los doce intermedios, de manera que estos datos los encontramos publicados desde los inicios de la serie hasta el final de los datos recuperados y digitalizados en 1944 con el Diario Español. La dirección del viento es una variable meteorológica que tiene un gran interés en el análisis del clima, y que sirve para la validación de otras variables.

Respecto a la velocidad del viento (VV), nos encontramos con muchos formatos distintos. En 1856 aparece la variable descrita con adjetivos (flojo, moderado, fuerte, vendaval). Es en 1879 cuando la variable pasa a tener carácter cuantitativo, publicándose probablemente en $\mathrm{m} / \mathrm{s}$, aunque no se dispone del metadato. A partir de 1890 y hasta 1916, la velocidad del viento se mide en los kilómetros recorridos en 24 horas. Y, finalmente, a partir de 1927, de nuevo se utilizan los adjetivos (calma, muy floja, floja, ventolina, moderado, algo fuerte, fuerte, Figura 9).

\subsection{Estado del cielo}

El estado del cielo presenta diferentes y muy variadas formas de interpretación y de anotación. En los inicios de la serie y hasta 1859 se utilizan diferentes adjetivos para definir el estado del cielo, como, por 
ejemplo, sereno, entresereno, nubecillas, nubes, nublado, entrecubierto, cubierto y otro tipo de adjetivos que se podrían incluir en los anteriores, como, por ejemplo, claro, celajes, toldado, casi cubierto, casi despejado, etc. Ya más adelante se pasa a definir el estado del cielo en décimas de cielo cubierto (1879-1881), para más tarde continuar otra vez con adjetivos (Figura 9) hasta el final de la serie en 1944.

Figura 9. Ejemplo de las observaciones de Viento y Estado del Cielo

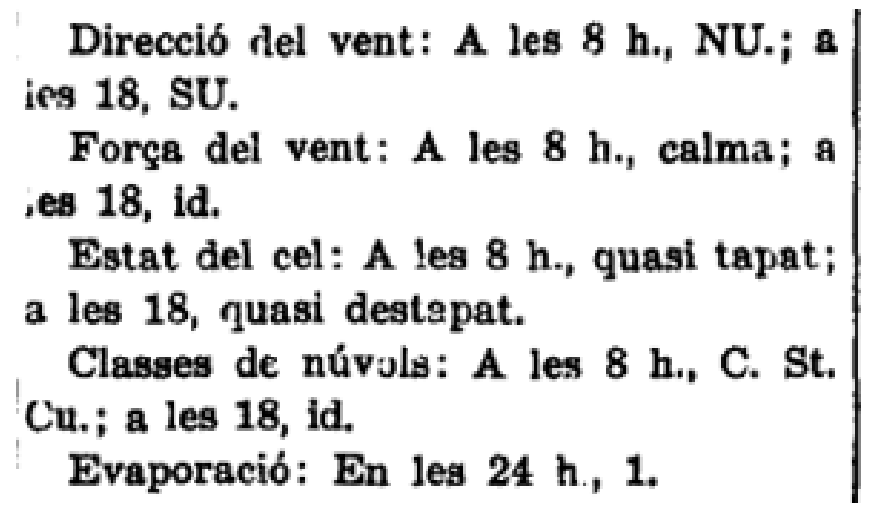

Fuente: La Llibertat (02/09/1936)

\section{DISCUSIÓN}

La prensa comarcal histórica es una buena fuente de información meteorológica y climática diferente a las fuentes de datos tradicionales. En el caso de la ciudad de Tarragona, se han podido recuperar numerosos datos meteorológicos diarios que hasta el momento no se disponía de ellos, y que, por tanto, se trata de información meteorológica y climática nueva y fresca, que nunca antes se había podido utilizar para la realización de estudios. A continuación se hará un repaso a las potencialidades y las carencias que presenta la prensa antigua digitalizada a la hora de extraer la información climática y un análisis de las variables meteorológicas del caso de Tarragona.

\subsection{Potencialidades}

- La prensa antigua digitalizada es una fuente de información meteorológica y climática muy importante y muy extensa, que permite recuperar períodos de información antiguos.

- La prensa digitalizada se encuentra en archivos públicos disponibles en internet, y por tanto cualquiera que quiera realizar algún estudio puede dedicarse a recuperar los datos que se publicaban en los diarios antiguos.

- La prensa histórica recoge un gran número de observaciones meteorológicas, tanto por la tipología como por la frecuencia. Así, se dispone de observaciones diarias como de observaciones a distintas horas, y también de observaciones de variables extremas a resolución diaria. Todo esto hace que se puedan recuperar hasta 3 observaciones, más los extremos diarios. Así es posible poder ver una buena evolución de posibles situaciones sinópticas.

- De este gran número de observaciones, encontramos también un gran número de variables meteorológicas, desde las más típicas como la temperatura y la precipitación, hasta la evaporación, o el estado del cielo, pasando por la presión atmosférica, o la dirección y velocidad del viento, entre otras.

- En caso de disponer de la fuente origina, la prensa histórica es un buen recurso para el relleno de lagunas, permitiendo disponer de un mayor porcentaje de datos recuperados.

- El metadato permite tener información extra de las variables meteorológicas. Por ejemplo, el metadato permite saber dónde se realizaban las medidas y disponer de la localización geográfica dentro de la ciudad. Por otro lado, el metadato es una ayuda para saber con qué instrumentos se medían las variables y en qué condiciones. 


\subsection{Carencias}

- La irregularidad temporal de la disponibilidad de información meteorológica es uno de los problemas que se ha detectado en la utilización de la prensa. Así pues, existen períodos en que se publicaba la información meteorológica, y en cambio períodos en que los ejemplares de los periódicos de la época se encuentran digitalizados pero no ofrecían la información meteorológica (grandes vacíos).

- Los fines de semana y festivos sobretodo los lunes no se publicaba el ejemplar de prensa, y como consecuencia nos encontramos con una pérdida de días en que seguramente los datos meteorológicos existían pero como no se publicaba el diario, esta información no se ha podido recuperar (vacíos puntuales y sistemáticos).

- La recurrencia de días con las mismas observaciones es otro problema que se encuentra en la prensa antigua. Este aspecto afecta a la serie ya que sólo se puede tener en cuenta el primer día de las observaciones, invalidando así los días siguientes, dejando vacíos de datos.

- Es necesario discriminar entre ejemplar de prensa antigua publicado digitalizado o publicado y no digitalizado, y, ejemplar de prensa no publicado y por tanto no digitalizado, para en un futuro saber si se podrá recuperar el dato meteorológico o no.

- Los datos no proceden siempre de la misma institución encargada de hacer la observación meteorológica, sino que hay épocas en que no se indica la procedencia de la información, que a menudo no se indica.

- El cambio en las unidades de medida es otro de los problemas para una buena homogeneización del dato meteorológico, y como se ha comentado anteriormente un metadato correcto es de vital importancia.

\subsection{Las variables meteorológicas}

Respecto a las variables meteorológicas, las más importantes a la hora de realizar estudios de cambio climático, que son la precipitación y las temperaturas máximas y mínimas, no empiezan a aparecer publicadas hasta el año 1879. Es a partir de esta fecha que la temperatura se empieza a medir en ${ }^{\circ} \mathrm{C}$ y la precipitación en mm hasta el final de la serie. Todo esto comporta que no se hayan de hacer conversiones y que la serie de datos sea más homogénea desde 1879. Añadir también que los vacíos de datos afectan especialmente a los totales de precipitación, ya que en el clima mediterráneo perder un día de datos (día que no se publicaba el diario) y si en ese día hubiera habido precipitación, puede afectar mucho a los totales mensuales y anuales de precipitación. En cambio en el caso de las temperaturas, estos vacíos de datos no afectan tanto a los promedios mensuales y anuales, y en casos muy extremos hasta se podría recuperar el dato con el promedio de los días anterior y posterior al vacío de datos, y disponer así de una serie mucho más completa a resolución diaria.

De las demás variables meteorológicas, destacamos la dirección y velocidad del viento, y la presión atmosférica. La presión atmosférica es una variable meteorológica que se publica a diferentes horas y aparece desde los inicios de la serie, con algunos cambios en la medida anteriormente comentados, pero que haciendo la conversión, se puede disponer de buenos datos de presión atmosférica, como ya se ha hecho en otras ciudades como la ciudad de Barcelona (Rodríguez et al., 1999). La dirección del viento la encontramos publicada también desde los inicios de la serie en 1809. Esto supone que se trate de una variable meteorológica importante para utilizar en estudios climáticos y poder observar diferentes comportamientos que haya podido sufrir la circulación atmosférica. Se puede utilizar también para la validación de datos de temperatura anómalos que se puedan encontrar con los controles de calidad. La velocidad del viento, al expresarse mayoritariamente con adjetivos se hace difícil saber cuál era la fuerza a la que se referirían cuando se utilizaba por ejemplo el viento moderado, ya que no se ha encontrado ningún metadato que definiera la magnitud del fenómeno.

Lo mismo pasa para las observaciones del estado del cielo. Estas se hacían a las mismas horas que las observaciones de presión atmosférica y la dirección del viento, pero en ningún momento se indica cuál es el criterio utilizado que se seguía para definir el estado del cielo con adjetivos. Así que el estado del cielo es un dato más que puede ser útil para validar otros datos, como por ejemplo la precipitación o las temperaturas cuando aparezcan dudas sobre éstas. El tipo de nubosidad en cambio no presenta ningún 
problema de interpretación, ya que, una vez se empiezan a anotar los datos, siempre se utiliza el mismo criterio de clasificación de nubes.

\section{CONCLUSIONES}

La actual preocupación por el cambio climático hace de gran necesidad disponer de registros meteorológicos instrumentales antiguos, para poder analizar la variabilidad del clima local y regional. Así pues la recuperación de los datos requiere de un buen análisis de la fuente de información.

Durante algunos períodos no hubo servicios meteorológicos oficiales, de manera que es interesante recuperar los datos de personas aficionadas que proporcionaban sus mediciones a la prensa escrita de la época, datos que son de gran interés climático en la actualidad.

La prensa histórica es una fuente de datos meteorológicos muy importante, que, con la digitalización de ejemplares y el posterior acceso público a estos datos a través de internet, hace posible que cualquier interesado en la meteorología y la climatología pueda dedicarse a recuperar los datos que se publicaban en los diarios antiguos, y así crear una buena base de datos digitalizados de distintas variables meteorológicas

En la actualidad existen muchos archivos y bibliotecas municipales que han escaneado y archivado en formato digital la prensa histórica y la han puesto a disposición del público general, de manera que esta posible base de datos puede ir aumentando y se pueden ir añadiendo distintos puntos de observación del país, de Europa y hasta del mundo.

El problema más importante que nos encontramos a la hora de rescatar datos meteorológicos antiguos a partir de la prensa histórica son los vacíos de datos que aparecen, en el caso de Tarragona el más importante desde 1812 a 1855, entre otros; también destacar los vacíos puntuales que corresponden con días festivos en los que no se publicaban los diarios. Otro problema son los metadatos. Durante algunos períodos no se dispone de información adicional de cuáles eran los aparatos con los que se realizaban las observaciones, hecho que puede dificultar el posterior tratamiento de variables meteorológicas.

Finalmente añadir que esto es un primer ensayo para el posterior análisis de las variables meteorológicas de la ciudad de Tarragona, que nos permitirá realizar controles de calidad a los datos y homogeneizar la fuente documental original, eliminando valores que ahora puedan resultar dudosos.

\section{BIBLIOGRAFIA}

BARRIENDOS, M. (1996-1997): "El clima histórico de Catalunya (siglos XIV-XIX). Fuentes, métodos y primeros resultados”, en Revista de Geografía, vol. XXX-XXXI, pp. 69-96.

BARRIENDOS, M.; GÓMEZ, B.; PEÑA, J.C. (1997): "Series meteorológicas instrumentales antiguas de Madrid y Barcelona (1780-1860). Características documentales y de observación", en Avances en climatología histórica en España. Martín Vide, J (Ed.). Oikos-Tau Barcelona, pp. 47-62.

BARRIENDOS, M. (1999): "La climatología histórica en el marco geográfico de la antigua monarquía hispana”, en Scripta Nova. Revista electrónica de geografía y ciencias sociales, Universidad de Barcelona, no 53 , pp. 1-34.

BARRIENDOS, M.; LLASAT, M.C. (2003): "The case of the 'Maldà' anomaly in the western mediterranean basin (AD 1760-1800): an example of a strong climatic variability" en Climatic Change, $\mathrm{n}^{\circ}$ 61, pp. 191-216.

BARRIENDOS, M. (2005): "Variabilidad climática y riesgos climáticos en perspectiva histórica. El caso de Catalunya en los siglos XVIII-XIX”, en Revista de historia moderna, n 23, pp. 11-34.

BRÁZDIL, R.; PFISTER, C.; WANNER, H.; VON STROCH, H.; LUTERBACHER, J. (2005): "Historical climatology in Europe - the state of the art", en Climatic Change, $n^{\circ} 70$, pp. 363-430.

CASTY, C.; WANNER, H.; LUTERBACHER, J.; ESPER, J.; BÖHM, R. (2005): "Temperature and precipitation variability in the european Alps since 1500", en International Journal of Climatology, $\mathrm{n}^{\mathrm{o}} 25$, pp. 1855-1880. 
DOMÍNGUEZ-CASTRO, F; SANTIESTEBAN, J.I.; BARRIENDOS, M.; MEDIAVILLA, R. (2008): "Reconstruction of drought episodes for central Spain from rogation ceremonias recorded at Toledo Cathedral from 1506 to 1900: A methodological approach", en Global and Planetary Change, n 63, pp. 230-242.

FERNÁNDEZ-MONTES, S.; RODRIGO, F.S. (2010): "Newspapers as early meteorological data sources in Andalusia (southern Spain), 1796-1830”, en EMS Annual Meeting Abstracts, vol.7, EMS2010-421.

LLASAT, M.C.; LLASAT-BOTIJA, M.; BARNOLAS, M.; LÓPEZ, L.; ALTAVA-ORTÍZ, V. (2009): "An analysis of the evolution of hydrometeorological extremes in newspapers: the case of Catalonia, 1982-2006", en Natural Hazards and Earth System Sciences, n 9, pp. 1201-1912.

LÓPEZ CORDERO, J.A. (2006): "La climatología en las fuentes documentales históricas giennenses", en Códice, no 19, pp. 31-44.

MARTín VIDE, J. (1992): El Clima. Geografia General dels Països Catalans. Enciclopèdia Catalana, Barcelona.

MARTÍN-VIDE, J.; BARRIENDOS, M. (1995): "The use of rogation ceremony records in climatic reconstruction: A case study from Catalonia (Spain)", en Climatic Change, $\mathrm{n}^{\mathrm{o}}$ 30, pp. 201-221.

NORDLI, P.Ø. (2001): "Reconstruction of nineteenth century summer temperatures in Norway by proxy data from farmers' diaries", en Climatic Change, n 48, pp. 201-218.

PAULING, A.; LUTERBACHER, J.; CASTY, C.; WANNER, H. (2006): "Five hundred years of gridded highresolution precipitation reconstructions over Europe and the connection to large-scale circulation", en Climate Dynamics, nº 26, pp. 387-405.

PIERVITALI, E.; COLACINO, M. (2001): "Evidence of drought in western Sicily during the period 15651915 from liturgical offices", en Climatic Change, n 49, pp. 225-238.

PROHOM, M. (2002): "El uso de los diarios de navegación como instrumento de reconstrucción climática. La marina catalana del siglo XIX", en Investigaciones Geográficas, n 28, pp. 89-104.

PROHOM, M.; HERRERO, M. (2008): "Hacia la creación de una base de datos climática de Cataluña (siglos XVIII a XXI)", en Tethys. Revista del tiempo y el clima del Mediterráneo occidental, no 5, pp. 3-11.

PROHOM, M.; BARRIENDOS, M.; AGUILAR, E.; RIPOLL, R. (2012): "Recuperación y análisis de la serie de temperatura diaria de Barcelona, 1780-2011", en Cambio climático. Extremos e impactos. Concepción Rodríguez-Puebla, Antonio Ceballos, Nube González-Reviriego, Enrique Morán-Tejeda y Ascensión Hernández-Encinas. Publicaciones de la Asociación Española de Climatología (AEC), Serie A, no 8. Salamanca, pp. 207-217.

PRZYBYLAK, R.; MAJOROWICZ, J.; WÓJCIK, G.; ZIELSKI, A.; CHORAZYCZEWSKI, W.; MARCINIAK, K.; NOWOSAD, W.; OLINSKI, P.; SYTA, K. (2005): "Temperature changes in Poland from the 16th to the 20th centuries", en International Journal of Climatology, n² 25, pp. 773-791.

RODRIGO, F.S.; ESTEBAN-PARRA, M.J.; CASTRO-DIEZ, Y. (1998): "On the use of the jesuit order prívate correspondence records in climate reconstructions: A case study from Castille (Spain) for 1634-1648 a.d.", Climatic Change, no 40, pp. 625-645.

RODRÍGUEZ, R.; BARRIENDOS, M.; PEÑA, J.C.; MARTÍN-VIDE, J. (1999): "Análisis de la serie de presión media mensual más larga de España: Barcelona (1780-1989)", en Asamblea Hipano-portuguesa de Geodesia y Geofísica, Universidad de Almería e IGN (formato CD-Rom) (ISBN;84-95172-10-0).

THORNDYCRAFT, V.R.; BARRIENDOS, M.; BENITO, G.; RICO, M.; CASAS, A. (2006): "The catastrophic floods of AD 1617 in Catalonia (northeast Spain) and their climatic context", en Hydrological SciencesJournal des Sciences Hydrologiques, no 51, pp. 899-912.

\section{BASE DE DATOS CONSULTADA}

http://www.tarragona.cat/lajuntament/conselleries/patrimoni/biblioteca-hemeroteca/premsadigitalitzada-1/premsa-digitalitzada 